\title{
hsa-miR-96 up-regulates MAP4K1 and IRS1 and may function as a promising diagnostic marker in human bladder urothelial carcinomas
}

\author{
YI WANG $^{1}$, HONGMEI LUO $^{2}$, YANGLE LI ${ }^{1}$, TIEDING CHEN ${ }^{1}$, SHENGPENG WU $^{1}$ and LUOYAN YANG ${ }^{1}$ \\ ${ }^{1}$ Department of Urology, The Second Xiangya Hospital of Central South University, Changsha, Hunan 410011; \\ ${ }^{2}$ Department of Histology and Embryology, University of South China, Hengyang, Hunan 421001, P.R. China
}

Received June 20, 2011; Accepted September 30, 2011

DOI: $10.3892 / \mathrm{mmr} .2011 .621$

\begin{abstract}
Numerous microRNAs (miRNAs) play crucial roles in cancer development. In this study, we report that hsa-miR-96 is expressed at higher levels in human bladder urothelial carcinomas compared to normal tissues. We found that hsa-miR-96 increased invasion and differentiation of human bladder T24 cells and promoted their growth. Down-regulation of hsa-miR-96 significantly affected the phenotype of bladder cancer T24 cells. The mRNA and protein levels of insulin receptor substrate 1 (IRS1) and MAP4K1 were significantly reduced in cells transfected with the hsa-miR-96 inhibitor when compared with levels in cells transfected with the empty plasmid vector or the negative control miRNA inhibitor. Altogether, these results suggest that hsa-miR-96 may affect the growth of bladder cancer cells by up-regulating IRS1 and MAP4K1 levels, functioning as a promising diagnostic marker in human bladder urothelial carcinomas.
\end{abstract}

\section{Introduction}

MicroRNAs (miRNAs) are a group of small (18-25 nt), non-coding RNA molecules found in the majority of eukaryotic cells, which may cause degradation of targeted mRNAs or inhibit their translation through binding to the targeted mRNAs specifically, and thus regulate post-transcriptional gene expression. miRNAs are capable of regulating proliferation and apoptosis of tumor cells, and are involved in the self-renewal and differentiation processes of cells. As biomarkers, miRNAs could be applied to cancer diagnosis, prognosis assessment and site-targeted therapy. Certain miRNA molecules could also be used as protocarcinogenic genes or tumor-suppressor genes to regulate tumors $(1,2)$. For example, expression levels of

Correspondence to: Professor Luoyan Yang, Department of Urology, The Second Xiangya Hospital of Central South University, Changsha, Hunan 410011, P.R China

E-mail: luoyany@yahoo.com

Key words: hsa-miR-96, diagnostic marker, MAP4K1, insulin receptor substrate 1
miR-34 (miRNA-34), miR-27a and miR-21 are all up-regulated in gastric cancers. miR-34 could inhibit the growth of gastric cancer cells with a mutation of p53. MiR-21 plays a role in the process of proliferation and apoptosis of gastric cancer cells, restrains expression levels of miR-27a and reduces the proliferation rate of gastric cancer cells. All of these findings are evidence that miRNAs are closely related to the occurrence and development of gastric cancer (3-5).

The 21-nt hsa-miR-96 (5'-aaucaugugcagugccaauaug-3') is located on human chromosome 7 . In the precursor of hsa-miR-96, a nucleotide polymorphism, which lies in the prosomatic stem-ring structure region, exists in a number of loci among various species. However, the mature sequence of hsa-miR-96 in species is somewhat conserved ( $>99 \%$ sequence similarity). For example, the mature miR-96 sequence of mice is completely homologous with that of humans (6).

MAP4K1, an activating factor upstream of the signaling pathway of stress-activated protein kinase (SAPK), has a structure similar to that of germinal center kinase (GCK), which includes four common sequences in the SH3 domain. It has been reported that these common sequences interact with the SH3 domain of MLK3 and MEKK1 (7). These genes are activated after being phosphorylated by cellular-Abelson (c-Abl), and then activate the pathway of Raf $\rightarrow$ MEK $1 \rightarrow$ MAPK/ ERK, therefore regulating the cell cycle, cell adhesion, migration and apoptosis (7).

Six members of the insulin receptor substrate family (IRS), named IRS1-IRS6, have been identified. They may play a significant role not only in the signaling pathway of cells but also in adjusting growth and functions of pancreatic $\beta$ cells $(8,9)$. Studies have shown that IRS3 and IRS4 alter the function of IRS1 and IRS2. When IRS1 and IRS2 are overexpressed, IRS3 and IRS4 limit the expression of IRS1 and IRS2 (10). Studies have also shown that IRS5 and IRS6 have just a few functions of signal transduction (11). IRS1 is the coding product of the gene IRS-1 (12), which widely exists in sensitive tissues of insulin, with a molecular weight of $185 \mathrm{kDa}$. Human IRS1 molecules have a PTB domain, a PH domain and more than 20 potential tyrosines (Tyr). Human IRS1 molecules also have over 50 Ser/Thy phosphorylation loci, which partly overlap with the gene sequence of Tyr and contribute to the coadjustment while phosphorylated. After pancreas is 
combined with the receptors, the subunit of receptor $\beta$ near the membrane-proximal region, through its Tyr90, phosphorylates itself. Then, the domain of IRS1 is combined with the region. IRS1 plays a significant role in the process where insulin and IGF-1 are transferring signals to intracellular signal pathways PI3K/Akt and ErkMAPK (13).

Research has revealed that hsa-miRNA-96 of MIA PaCa-2 and PANC-1 pancreatic cancer cell lines regulate the KRAS gene and weaken the invasive ability and migration of cell lines, thus functioning as a cancer-suppressor gene (14). High expression of hsa-miR-96 has previously been found in bladder cancer tissues (data not shown). However, the mechanism of hsa-miR-96 in bladder urothelial carcinoma remains unclear.

In this study, we found that down-regulation of hsa-miR-96 significantly affects the phenotype of bladder cancer T24 cells. Furthermore, the mRNA and protein levels of IRS1 and MAP4K1 were significantly reduced in cells transfected with the hsa-miR-96 inhibitor when compared with levels in cells transfected with the empty plasmid vector or the negative control miRNA inhibitor. These results suggest that hsa-miR-96 may affect the growth of bladder cancer cells by up-regulating IRS1 and MAP4K1 levels, functioning as a promising diagnostic marker in human bladder urothelial carcinomas.

\section{Materials and methods}

Tissue samples and cancer cell lines. Fresh bladder cancer tissues and normal bladder tissues adjacent to carcinomas were collected at surgery and immediately stored in liquid nitrogen at $-80^{\circ} \mathrm{C}$. Bladder cancer cell lines T24 and 5637 were purchased from Shanghai GenePharma Technology Co., Ltd (Shanghai, China). All experiments were conducted according to the ethical guidelines of the Central South University and of the University of South China. Patient consent was also obtained.

Cell lines and target genes tested. Software including miRBase, TargetScan and microRNA were used to analyze the target genes of hsa-miR-96 (IRS1, MAP4K1, MAP2K2, MAP3K3 and MAP4K4). Reverse-transcription PCR was performed to detect the levels of these genes in cells. Three target genes and cell lines with appropriate expression levels were selected for subsequent experiments.

Real-time PCR and Western blotting. Total RNAs were extracted from fresh tissues or cells with TRIzol reagent (Invitrogen, Carlsbad, CA, USA). Real-time PCR assays were performed to detect the expression levels of hsa-miR-96 and 3 target genes in bladder cancer and normal bladder tissues. Real-time PCR assays were performed using an miRNeasy mini kit (Qiagen, USA; cat no. 217004) and an miRNA Q-PCR detection kit (GeneCopoeia, Rockville, MD; USA, cat no. R0101L). RNA loading was normalized to the level of human U6 small nuclear RNA (snRNA). The primers used in this study were designed using software Primer 5 and are shown in Table I.

Western blotting was performed for detection of target proteins. The primary antibodies used were as follows: IRS1 (cat no. sc-559; Santa Cruz Biotechnology, Santa Cruz, CA, USA), MAP4K1 (cat no. ab2646; Abcam, Cambridge, UK),
Table I. List of oligonucleotides.

\begin{tabular}{ll} 
Oligonucleotides & \multicolumn{1}{c}{$\begin{array}{c}\text { Primer sequences } \\
\left(5^{\prime}-3^{\prime}\right)\end{array}$} \\
\hline Homo-IRS1-F & ACTCTCATGTCTTGCCTCAC \\
Homo-IRS1-R & GTGTGCTTAAAGGATCTTGG \\
Homo-MAP4K1-F & TCTTTAAGGCTCGAGACAAG \\
Homo-MAP4K1-R & GCCTGTCACTTGGTAGATGT \\
Homo-MAP2K2-F & CCTGGACTATATTGTGAACGA \\
Homo-MAP2K2-R & AAAATCCACTTCTTCCACCT \\
Homo-MAP3K3-F & GGTCACTCAAATAGGCAGAG \\
Homo-MAP3K3-R & CTTTTCATGCTTGAGCTTCT \\
Homo-MAP4K4-F & TGCCTGGTGAGTCTACTCTT \\
Homo-MAP4K4-R & TCCTTTGTTGCTCTTCTAGC \\
Homo-GAPDH-F & ACCACAGTCCATGCCATCAC \\
Homo-GAPDH-R & TCCACCACCCTGTTGCTGTA \\
Hsa-miR-96-F & TTTGGCACTAGCACATT \\
Hsa-miR-96-R & TTTGGCACTAGCACATT \\
Human U6 snRNA-F & CTCGCTTCGGCAGCACA \\
Human U6 snRNA-R & AACGCTTCACGAATTTGCGT \\
Hsa-miR-96 inhibitor & AGCAAAAAUGUGCUAGUGCCAAA \\
Control miRNA inhibitor & CAGUACUUUUGUGUAGUACAA \\
\hline
\end{tabular}

IRS1, insulin receptor substrate 1 .

MAP2K2 (cat no. 11049-1-AP; PTGlab) and glyceraldehyde 3-phosphate dehydrogenase (GAPDH) (cat no. Mab-2005079; ProMab). Each experiment was repeated more than 3 times.

Cell transfection. hsa-miR-96 and the control miRNA inhibitors were transfected into cells using Lipofectamine 2000 (Invitrogen). hsa-miR-96 and control miRNA inhibitors were purchased from Shanghai GenePharma Technology Co., Ltd. and are shown in Table I.

Cell growth, apoptosis, cell migration and cell invasion assays. Cell growth was determined at 6, 12, 24 and $48 \mathrm{~h}$ following T24 cell transfection using MTT. Cell apoptosis was detected for T24 cells at $48 \mathrm{~h}$ following transfection by AV/PI and FCM. Cell invasion assays were performed to detect the ability of cells passing through a Transwell chamber (Corning, NY, USA). Cells were seeded on a Matrigel-coated membrane matrix in the insert of a 6 -well cultured plate $48 \mathrm{~h}$ following transfection, and fetal bovine serum (FBS) was added to the lower chamber. After being cultured for $24 \mathrm{~h}$, the lower surface of the Transwell chamber was observed by the inverted microscope (Olympus), and numbers of cells were counted.

Statistical analysis. Data analyses were performed by one-way LSD test or Student's t-test, respectively, using the SPSS 16.0 program. $\mathrm{P}<0.05$ was considered to be statistically significant.

\section{Results}

Higher expression levels of hsa-miR-96 in bladder urothelial carcinomas than in normal tissues. In order to determine 


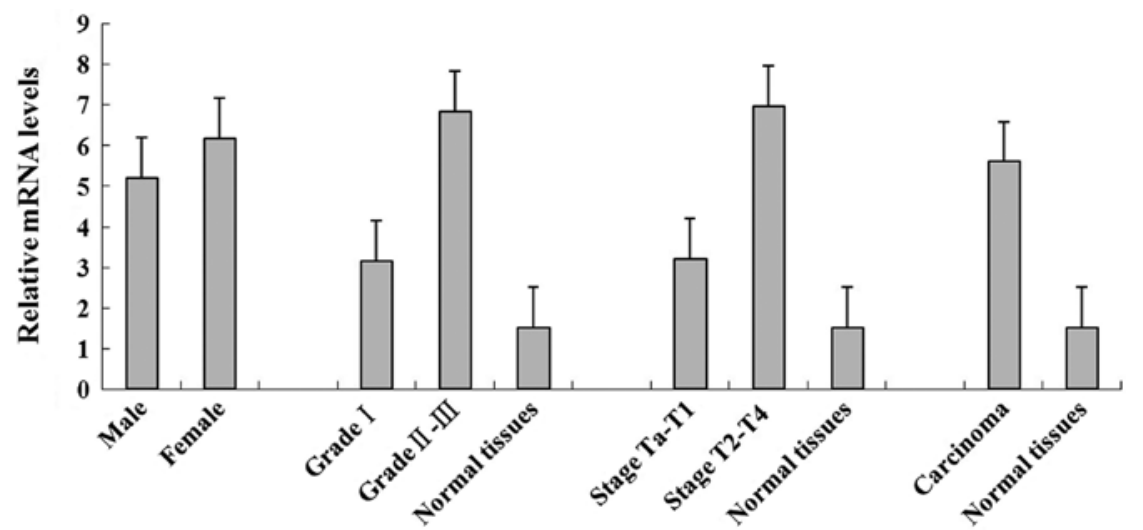

Figure 1. Expression of hsa-miR-96 in bladder urothelial carcinomas. Real-time PCR was performed to determine the expression levels of hsa-miR-96 in bladder urothelial carcinomas and normal tissues. RNA input was normalized to human U6 snRNA. Experiments were repeated more than 3 times. The comparative $\mathrm{Ct}\left(\Delta \Delta \mathrm{C}_{\mathrm{t}}\right)$ method was used to analyze results, and the data are presented as the means $\pm \mathrm{SD}$. $\mathrm{P}<0.01$ indicates statistical significance when compared to the normal bladder tissues.

whether hsa-miR-96 levels in bladder urothelial carcinomas are different from those in the normal tissues, real-time PCR was performed in 33 cases of bladder urothelial carcinomas and 9 cases of adjacent normal bladder tissues. The comparative $\mathrm{Ct}\left(\Delta \Delta \mathrm{C}_{\mathrm{t}}\right)$ method was used to determine the fold-difference in hsa-miR-96 expression in the various bladder urothelial carcinoma tissues relative to the adjacent normal bladder tissues.

As shown in Fig. 1, the expression of hsa-miR-96 was much higher in bladder urothelial carcinoma than in normal bladder tissues (Student's t-test, $\mathrm{P}<0.01$ ). Moreover, analyses of hsa-miR-96 expression in tumors of various clinical stages and pathological classifications demonstrated that hsa-miR-96 expression was higher in T2-T4 stage than in Ta-T1 stage tumors. Furthermore, hsa-miR-96 expression was higher in pathological grade II-III than in grade I disease (one-way LSD test, $\mathrm{P}<0.01)$. However, there was no significant difference in hsa-miR-96 expression levels between tissues from male and female patients (Student's test, $\mathrm{P}>0.05$ ). These results suggest that hsa-miR-96 is expressed in bladder urothelial carcinomas at a much higher level than in normal tissues.

Expression levels of the target genes of hsa-miR-96 in cultured cell lines. Using software including miRBase, TargetScan and MicroRNA, the putative and reported target genes of hsa-miR-96 were extensively analyzed. Among the target genes of hsa-miR-96, IRSI and MAP3K genes (MAP4K1, MAP2K2, MAP3K3 and MAP4K4) were selected for further investigation in the bladder cancer cell lines T24 and 5936. The expression of target genes in these two cell lines was determined by reverse-transcription PCR.

As shown in Fig. 2, the results revealed that the relative $R$ values of IRS1, MAP4K1 and MAP2K2 were higher in bladder cancer cell line T24 than in 5637 cells. To detect the expression levels of hsa-miR-96 in bladder cancer cell lines T24 and 5637, real-time PCR was also conducted. Our real-time PCR results showed that the average levels of hsa-miR-96 were higher in T24 than in 5936 cells (Student's t-test, $\mathrm{P}<0.01$ ) (data not shown). For easy detection, T24 cells were used for further study.

Effect of hsa-miR-96 down-regulation on bladder cancer T24 cells. To study the effects of hsa-miR-96 on bladder cancer T24

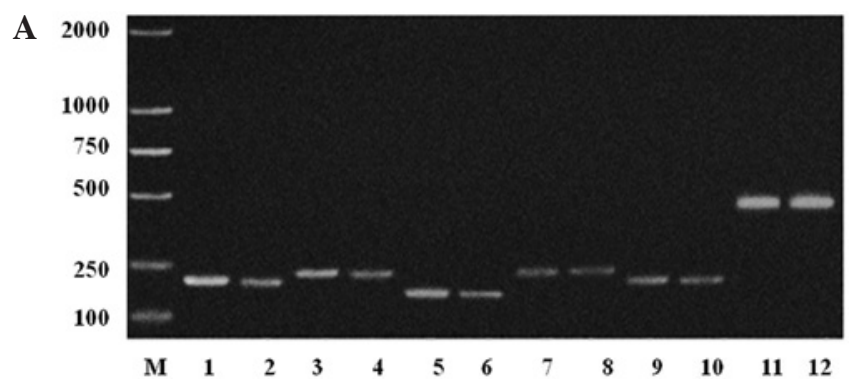

B

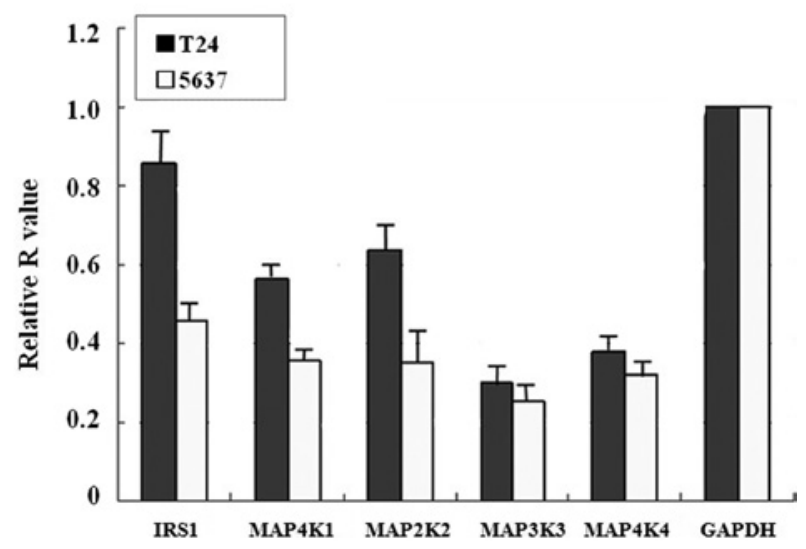

Figure 2. (A) Expression levels of a number of hsa-miR-96 target genes in two cell lines (T24 and 5637) as detected by reverse-transcription PCR. Lane 1, IRS1 in T24 cells; lane 2, IRS1 in 5637 cells; lane 3, MAP4K1 in T24 cells; lane 4, MAP4K1 in 5637 cells; lane 5, MAP2K2 in T24 cells; lane 6, MAP2K2 in 5637 cells; lane 7, MAP3K3 in T24 cells; lane 8, MAP3K3 in 5637 cells; lane 9, MAP4K4 in T24 cells; lane 10, MAP4K4 in 5637 cells; lane 11, GAPDH in T24 cells; and lane 12, GAPDH in 5637 cells. The integral optical density (IOD) was determined. (B) Graphic presentation of data obtained from panel A and two additional independent experiments. The signals in each lane in panel A were quantified and the ratio of each target gene to GAPDH levels was calculated. GAPDH was used as a housekeeping control. Data are shown as the mean $\pm \mathrm{SD}$.

cells, hsa-miR-96 was down-regulated by transfecting cells with the hsa-miR-96 or the negative control miRNA inhibitors (Table I) using Lipofectamine 2000. The experiments were designed as follows: Group a, cells transfected with the empty plasmid vector; group $b$, cells transfected with the negative 
A

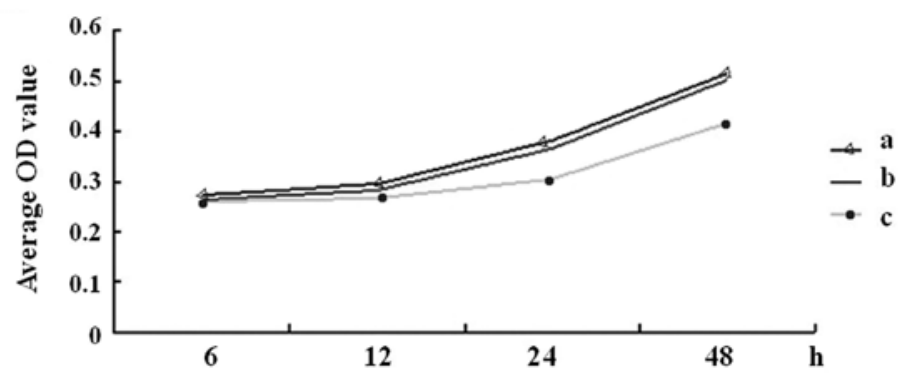

B

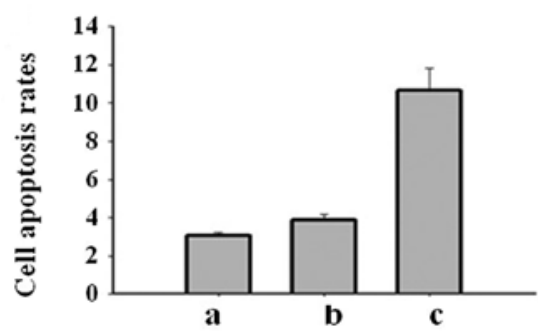

C

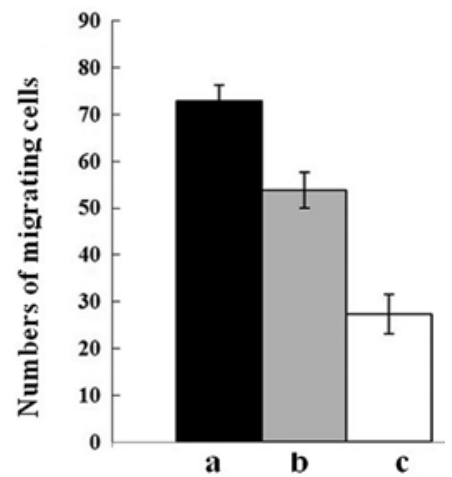

Figure 3. (A) Cell growth curves were constructed as determined using the MTT assay. Data are presented as the average OD value. (B) Comparison of cell apoptosis rates in the different groups detected by AV/PI and FCM. Data are presented as the means \pm SD (one-way LSD test; P<0.01). (C) Comparison of the number of migrating cells in the 3 groups in the cell invasion experiment. Data are presented as the means \pm SD (one-way LSD test; P<0.01). Group a, cells transfected with the empty plasmid vector; group b, cells transfected with the negative control miRNA inhibitors; and group c, cells transfected with the hsa-miR-96 inhibitor.

A

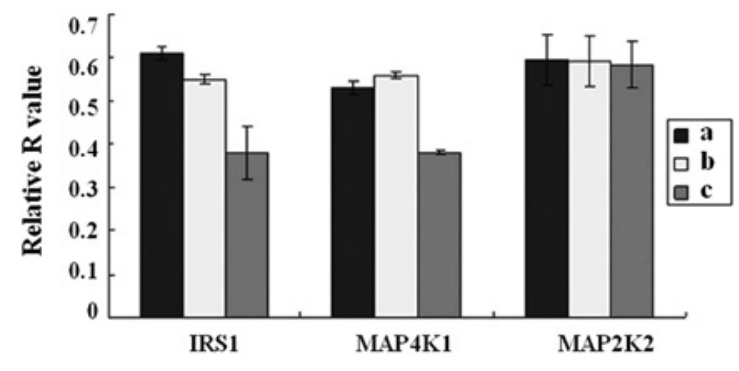

B

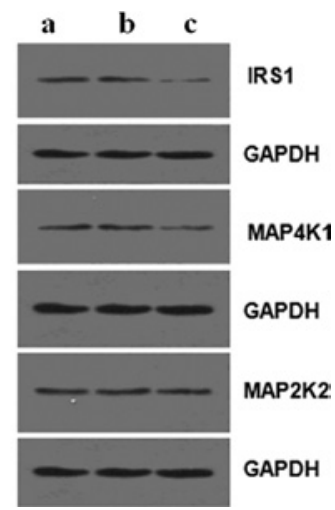

Figure 4. Levels of 3 hsa-miR-96 target genes were determined in cells transfected with the hsa-miR-96 inhibitor (group c), empty plasmid vector (group a) or the negative control miRNA inhibitor (group b). The GAPDH gene was used as the control. (A) RNA expression levels of these target genes were detected by real-time PCR. (B) Protein expression levels of the 3 target genes were detected by Western blotting.

control miRNA inhibitors; and group c, cells transfected with the hsa-miR-96 inhibitor.

Cell growth was examined by the MTT method at 6, 12, 24 and $48 \mathrm{~h}$ post-transfection. Data from the 3 groups (group a, b, and c) are shown with the average OD value. The results in Fig. 3A showed that the growth of cells transfected with the hsa-miR-96 inhibitor was significantly lower compared to the growth of cells transfected with the empty plasmid vector or the negative control miRNA inhibitors (one-way LSD test $\mathrm{P}<0.01)$. The apoptosis assay was performed by the AV/PI and FCM method for the 3 groups. The apoptosis rate of cells transfected with the hsa-miR-96 inhibitor was higher than that in cells transfected with the empty plasmid vector or the negative control miRNA inhibitors (one-way LSD test; $\mathrm{P}<0.01$ ) (Fig. 3B). Fig. 3C showed that the number of migrating cells in the group transfected with the hsa-miR-96 inhibitor was significantly lower compared to those of the cells transfected with the empty plasmid vector or the negative control miRNA inhibitors (one-way LSD test; $\mathrm{P}<0.01$ ). Altogether, these results suggest that hsa-miR-96 down-regulation significantly affects the phenotype of bladder cancer T24 cells.

Down-regulation of hsa-miR-96 decreases the $m R N A$ and protein levels of IRS1 and MAP4K1. As mentioned previously, the target genes of hsa-miR-96, including IRS1 and MAP4K1, are involved in the growth of carcinoma cells. In order to understand how hsa-miR-96 affects the phenotype of bladder cancer T24 cells, cells were transfected with the hsa-miR-96 inhibitor, the empty plasmid vector or the negative control miRNA inhibitor. The total RNA and proteins were extracted. 
Real-time PCR and Western blotting were performed to detect the RNA and protein levels of the hsa-miR-96 target genes.

As shown in Fig. 4A, the mRNA levels of IRS1 and MAP4K1 were significantly reduced in cells transfected with the hsa-miR-96 inhibitor when compared with those in cells transfected with the empty plasmid vector or the negative control miRNA inhibitor (one-way LSD test, $\mathrm{P}<0.01$ ). However, the mRNA expression levels of MAP2K2 were similar among the 3 groups (one-way LSD test, $\mathrm{P}>0.05$ ).

As shown in Fig. 4B, the protein levels of IRS1 and MAP4K1 were significantly reduced in cells transfected with hsa-miR-96 inhibitor when compared with levels in cells transfected with the empty plasmid vector, or the negative control miRNA inhibitor (one-way LSD test, $\mathrm{P}<0.01$ ). However, the protein levels of MAP2K2 were similar among the 3 groups (one-way LSD test, $\mathrm{P}>0.05$ ). Altogether, these results suggest that hsa-miR-96 may affect growth of bladder cancer T24 cells by up-regulating IRS1 and MAP4K1 levels.

\section{Discussion}

Studies have shown that miRNAs regulate proliferation and apoptosis of cells, and play a role in cell differentiation. Certain miRNAs have been used to regulate the occurrence and development of tumors. Si et al (15) demonstrated the growth of breast cancer MCF-7 cells and the ability to develop a tumor in nude mice was inhibited in vivo by transfecting miRNA (antisense oligonucleotide suppressant) into MCF-7 cells. Chan et al (16) found that the miR-21 inhibitor could promote the apoptosis of human colloid metrocyte tumor cells and restrain cell proliferation. It has also been discovered that the expression of miR-21 was up-regulated in solid tumors by analyzing the miRNA expression profile of solid tumors $(17,18)$.

Yu et al (14) found that the expression level of miR-96 in pancreatic cancer tissues was consistently lower compared to that in normal pancreatic tissues. However, Lin et al (19) found that miR-96 expression in breast cancer cells and tissues is markedly higher compared to that in normal breast epithelial cells and tissues. While exploring the diagnostic marker in human bladder urothelial carcinoma, Yamada et al (20) found that miR-96 and miR-183 expression in urine could serve as a tumor marker for human bladder urothelial carcinomas. Furthermore, combined with urine exfoliative cytology examination, miR-96 could serve as a diagnostic marker for bladder tumors.

In this study, real-time PCR was performed to detect hsa-miR-96 expression in bladder urothelial carcinoma, and we demonstrated that hsa-miR-96 expression in bladder urothelial carcinoma was higher compared to that in normal bladder tissues. Furthermore, we discovered that hsa-miR-96 expression in the superficial tumors was lower than that in muscularis invasive tumors, and tightly related to the pathological classification and clinical stages of bladder urothelial carcinoma. These results showed that hsa-miR-96 may be involved in the process of occurrence, development and infiltration of bladder urothelial carcinomas. Our results also showed that the hsa-miR-96 inhibitor inhibits the growth of cells and the ability of cellular invasion and promotes apoptosis, suggesting that hsa-miR-96 may regulate the biological behaviors of bladder cancer.
In the study of the effect of hsa-miR-96 on the signaling pathway of bladder cancer, real-time PCR and Western blotting were employed to detect the expression of MAP4K1. The expression of MAP4K1 was lower compared to groups $a$ and $b$ (normally developed cells and negative control of microRNA, respectively). hsa-miR-96 may up-regulate MAP4K1 expression. MAP4K1 could be activated after being phosphorylated by tyrosine kinase and in turn could activate the $\mathrm{Raf} \rightarrow \mathrm{MEK} 1 \rightarrow \mathrm{MAPK} /$ ERK pathway so as to modulate the cell cycle and cell adhesion, migration and apoptosis (7). In bladder cancer, although the expression of MAP4K1 is up-regulated, the process of its phosphorylation may be inhibited, thus blocking the MAPK/ERK pathway.

There are two characteristic signaling pathways modulated by insulin: the MAPK pathway and PI3K cascade. The MAPK pathway mainly modulates the growth and differentiation of cells, while the PI3K cascade plays a role in adjusting insulin functions and insulin-involved cell metabolism. The tyrosine phosphorylation of ISR1 interacts with subunit p85, which is regulated by PI3K, thus activating PI3K. After being phosphorylated, Akt may be activated by PIP3. Akt phosphorylation is crucial in the anabolism and catabolism of organisms. However, the serine phosphorylation of IRS1 may reduce the activity of IRS1 (21). Yang et al (22) discovered that overexpression of SOCS3 (a suppressor of cytokine signaling-3) in pig adipocytes inhibits the function of insulin signal peptide. The inactivation of IRS1 promotes the metastasis of breast cancer and the PI3K/Akt signaling pathway was inhibited (23).

To assess the expression of IRS1, real-time PCR and Western blotting were conducted in the 3 cell groups. IRS1 expression was lower in the group transfected with hsa-miR-96 inhibitor compared to the untreated and negative control groups (groups c, a and b, respectively). hsa-miR-96 up-regulated the expression of IRS1. However, in the group treated with the hsa-miR-96 inhibitor, the apoptosis of cancer cells increased while the invasive ability decreased. Therefore, it is possible that hsa-miR-96 up-regulates IRS1 expression, facilitates the serine phosphorylation of IRS1, blocks the PI3K/ Akt signal transduction pathway and leads to the metastasis of cancer cells. MAP2K2 expression was similar in all 3 groups, indicating that MAP2K2 could not serve as a target gene of hsa-miR-96 in bladder cancer.

\section{Acknowledgements}

This study was supported by grants from the Educational Commission Fund of Hunan Province in China (no. 10C1164).

\section{References}

1. Waldman SA and Terzic A: MicroRNA signatures as diagnostic and therapeutic targets. Clin Chem 54: 943-944, 2008.

2. Fabbri M, Croce CM and Calin GA: MicroRNAs. Cancer J 14: $1-6,2008$

3. Chan $\mathrm{SH}, \mathrm{Wu} \mathrm{CW}, \mathrm{Li} \mathrm{AF}$, et al: miR-21 microRNA expression in human gastric carcinomas and its clinical association. Anticancer Res 28: 907-911, 2008.

4. Liu T, Tang H, Lang Y, et al: MicroRNA-27a functions as an oncogene in gastric adenocarcinoma by targeting prohibitin. Cancer Lett 273: 233-242, 2009.

5. Ji Q, Hao X, Meng Y, et al: Restoration of tumor suppressor miR-34 inhibits human p53-mutant gastric cancer tumorspheres. BMC Cancer 8: 266, 2008. 
6. Mourelatos Z, Dostie J and Paushkin S: miRNPs: a novel class of ribonucleoproteins containing numerous microRNAs. Genes Dev 16: 720-728, 2002.

7. Oehrl W, Kardinal C and Ruf S: The germinal center kinase (GCK)-related protein kinases HPK1 and KHS are candidates for highly selective signal transducers of Crk family adapter proteins. Oncogene 17: 1893-1901, 1998.

8. Castagnola P and Giaretti W: Mutant KRAS, chromosomal instability and prognosis in colorectal cancer. Biochim Biophys Acta 1756: 115-125, 2005.

9. Lee J, Jang KT and Ki CS: Impact of epidermal growth factor receptor (EGFR) kinase mutations, EGFR gene amplifications, and KRAS mutations on survival of pancreatic adenocarcinoma. Cancer 109: 1561-1569, 2007.

10. Sesti G, Federici M and Hribal ML: Defects of the insulin receptor substrate (IRS) system in human metabolic disorders. FASEB J 15: 2099-2111, 2001.

11. Kulkarni RN, Winnay JN and Daniels M: Altered function of insulin receptor substrate-1-deficient mouse islets and cultured beta-cell lines. J Clin Invest 104: R69-R75, 1999.

12. Balakrishnan S, Kumpatla S and Viswanathan V: Comparative analysis of transcription factors of insulin signaling. Indian J Biotech 9: 24-30, 2010.

13. Taniguchi CM, Emanuelli B and Kahn CR: Critical nodes in signalling pathways: insights into insulin action. Nat Rev Mol Cell Biol 7: 85-96, 2006.

14. Yu S, Lu Z and Liu C: miRNA-96 suppresses KARS and functions as a tumor suppressor gene in pancreatic cancer. Cancer Res 70 6015-6025, 2010.
15. Si ML, Zhu S and Wu H: miR-2l-mediated tumor growth. Oncogene 26: 2799-2803, 2007.

16. Chan JA, Krichevsky AM and Kosik KS: MicroRNA-21 is an antiapoptotic factor in human glioblastoma cells. Cancer Res 65: 6029-6033, 2005.

17. Lu J, Getz G and Miska EA: MicroRNA expression profiles classify human cancers. Nature 435: 834-838, 2005.

18. Volinia S, Calin GA and Liu CG: A microRNA expression signature of human solid tumors defines cancer gene targets. Proc Natl Acad Sci USA 103: 2257-2261, 2006.

19. Lin H, Dai T and Xiong H: Un-regulated miR-96 induces cell proliferation in human breast cancer by down-regulating transcriptional factor FOXO3a. PLoS One 5: e15797, 2010.

20. Yamada Y, Enokida H and Kojima S: MiR-96 and miR-183 detection in urine serve as potential tumor markers of urothelial carcinoma: correlation with stage and grade, and comparison with urinary cytology. Cancer Sci 102: 522-529, 2011.

21. Hei YJ: Recent progress in insulin signal transduction. J Pharmacol Toxicol Methods 40: 123-135, 1998.

22. Yang SJ, Xu CQ and Wu JW: SOCS3 inhibits insulin signaling in porcine primary adipocytes. Mol Cell Biochem 345: 45-52, 2010.

23. Ma Z, Gibson SL and Byrne MA: Suppression of insulin receptor substrate 1 (IRS-1) promotes mammary tumor metastasis. Mol Cell Biol 26: 9338-9351, 2006. 